\title{
La innovación en la enseñanza de alemán realizada por Richard Ratti-Kámeke (1873-1945) en Barcelona a principios del s. xx
}

\author{
Bernd Marizzi ${ }^{1}$
}

Recibido: 21 de julio de 2020 / Aceptado: 3 de junio de 2021

Resumen. El objetivo de este artículo, que aborda un punto ciego en la historia del aprendizaje de idiomas en España, es doble: detallar información sobre uno de los autores y enseñantes del alemán —en este caso el berlinés Richard Ratti-Kámeke (18731945) - y a la vez detallar aspectos de la innovación en la enseñanza del alemán entre hablantes del español llevada a cabo por él. En este sentido, el estudio se integra en AILA Research Network for the History of Language Learning and Teaching (HoLLT. net) cuyo objetivo es fomentar la investigación sobre la historia del aprendizaje y la enseñanza de idiomas sobre una base amplia e internacional.

Palabras clave: Enseñanza de lengua alemana; innovación; 1916-1945; España; Richard Ratti-Kámeke (1873-1945).

[en] The innovation in the teaching of German by Richard Ratti-Kámeke (1873-1945) in Barcelona in the early XXth century

Abstract. The aim of this article, which addresses a blind spot in the history of language learning in Spain, is twofold: to detail information about one of the authors and teachers of German — in this case the Berliner Richard Ratti-Kámeke (1873-1945) — and at the same time detail aspects of the innovation in the teaching of German among Spanish speakers carried out by him. In this sense, the study is integrated into AILA Research Network for the History of Language Learning and Teaching (HoLLT.net) whose objective is to promote research on the history of language learning and teaching on a broad and international basis.

Keywords: Teaching German; Innovation; 1916-1945; Spain; Richard Ratti-Kámeke (1873-1945).

[fr] Le renouveau de l'enseignement de l'allemand mis en place par Richard Ratti-Kámeke (18731945) à Barcelone au début du XXe siècle

Résumé. L'objectif de cet article, qui traite d'un point aveugle dans l'histoire de l'apprentissage des langues en Espagne, est double: fournir des informations détaillées sur un auteur de manuels et professeur d'allemand estimé - le Berlinois Richard Ratti-Kámeke (1873-1945) — et en même temps détailler certains aspects du renouveau que lui doit l'enseignement de l'allemand chez les locuteurs hispanophones. L'étude s'inscrit ainsi dans le cadre des travaux du réseau de recherche AILA pour l'histoire de l'apprentissage et de l'enseignement des langues (HoLLT.net), qui vise à promouvoir la recherche sur l'histoire de l'apprentissage et de l'enseignement des langues sur une base large et internationale.

Mots-clés: Enseignement de la langue allemande; innovation; 1916-1945; Espagne; Richard Ratti-Kámeke (1873-1945).

Sumario: 1. Introducción. 2. Ratti-Kámeke. 3. Innovaciones. 4. Alemán como lengua de la ciencia. 5. Conclusión. 6. Bibliografía.

Cómo citar: Marizzi, Bernd (2021). La innovación en la enseñanza de alemán realizada por Richard Ratti-Kámeke (1873-1945) en Barcelona a principios del s. xx. Didáctica. Lengua y Literatura, 33, 71-81.

\section{INTRODUCCIÓN}

Una de las maneras de seguir las huellas que deja la actividad docente es investigar sobre los materiales que se emplean en ella, y a este respecto se observa el curioso fenómeno de que los libros escolares o libros de texto no gozan del mismo prestigio y de la misma estima que otras formas de libros impresos. Hasta hace poco los libros de

\footnotetext{
Universidad Complutense de Madrid, Dpto. Filosofía y Sociedad marizzi@ucm.es
} 
texto, aun siendo tradicionalmente uno de los elementos pedagógicos más relevantes de la educación reglada, estaban considerados como objetos sin valor y de carácter fungible. En España apenas existían centros dedicados a la recopilación y al estudio del material educativo. Pero en honor a la verdad, ha de decirse que en los últimos decenios ha aumentado el interés por los libros de texto y los manuales escolares antiguos. ${ }^{2}$ La idea básica que llevó a esta mejora fue que se debía recurrir a los instrumentos pedagógicos como fuentes para el estudio del curriculum y de los métodos de enseñanza de cada época. Pero los libros de texto también pueden ser fuente de otras informaciones: no solo transfieren conocimientos, sino también los valores de una sociedad, y son decisivos en el proceso de trasmitir ciertas ideas de los grupos dominantes.

En este contexto tampoco hay que olvidar que la enseñanza deja sus huellas en la mente de todos, y que los libros escolares y de texto han llegado al habla popular, como por ejemplo en la expresión castellana tiene más cuento que Calleja, que hace referencia a Saturnino Calleja Fernández (1853-1915). ${ }^{3}$ Cuando a mediados del s. XIX se extendió entre las clases formadas la necesidad de aprender idiomas extranjeros, adquirió gran popularidad un sistema de aprendizaje ideado por el alemán Johann Franz Ahn (1796-1865) que se aplicó para estudiar idiomas como el francés, el inglés, el italiano y también el alemán. Tal fue el éxito de este método que ya a finales del s. XIX se propagó la expresión según el método de Ahn "para indicar que algo o alguien es cómico, incongruente o inservible. Se usa con sarcasmo, para ridiculizar algo o a alguien"4.

Hoy en día se sigue utilizando la expresión por el método Ollendorff para indicar que el diálogo entre políticos se reduce a que uno emite frases que nada tienen que ver con el tema que su interlocutor pretende tratar. Es clara la referencia al sistema de enseñanza de lenguas vivas propagado a principios del s. XIX por el lingüista alemán Heinrich Georg Ollendorff (1803-1862) y que fue popularizado en España por Eduardo Benot (1822-1907), autor de un gran número de obras de enseñanza, entre otras de una gramática alemana.

La investigación en la historia de la enseñanza de los idiomas modernos puede aportar valiosas informaciones no solo acerca de la metodología y otros campos más técnicos de la didáctica de las lenguas así como acerca de los profesores implicados, sino también sobre otros aspectos como la historia de la construcción de la imagen de los germanoparlantes y sus respectivos países a través de los manuales.

Un resultado de estas investigaciones es la bibliografía comentada del aprendizaje de la lengua alemana en la península ibérica llevada a cabo por un grupo de germanistas portugueses y españoles. En Deutschlernen in Spanien und Portugal. Eine teilkommentierte Bibliographie von 1502 bis 1975 se recogen los libros de texto y diccionarios de alemán que se produjeron o utilizaron en España y Portugal entre 1502 y mediados de los años 70 del siglo pasado (Marizzi, Cortez y Fuentes Morán, 2018). Estos materiales se clasifican y describen de acuerdo con un registro analítico común donde se trata de los avances en la metodología empleada en ellos.

\section{RICHARD RATTI-KÁMEKE (1873-1945)}

Una de las personas que destacó en la innovación de la enseñanza del alemán y sus materiales fue al mismo tiempo uno los autores de libros de texto de lengua alemana más prolíficos en España, Richard Ratti-Kámeke (1873-1945), cuya actividad le llevó desde Berlín primero a Nápoles y luego a Barcelona. En esta ciudad, además de mantener una academia de lenguas, fue autor y editor de la Biblioteca Ratti, que contaba con una serie de manuales de alemán, inglés, francés y hasta de japonés. Pero sobre todo se centraba en la enseñanza del alemán como lengua científica, produciéndose en este contexto una serie de innovaciones didácticas que se detallarán en el punto 3.

Pero antes se indicarán brevemente los datos más importantes de la vida de Richard Ratti-Kámeke. Nacido el 11 de agosto de 1873 en Berlín como Robert Richard Stephan Ratti, hijo de Robert Ratti y de Anna Axeline Hermine Ratti (de soltera Kameke), fue bautizado según el rito protestante el 31 de septiembre en la St. Petri-Kirche. En el listado de comerciantes de Berlín, su padre aparece en 1873 como Galanteriewarenhändler (comerciante de bisutería), empadronado en el tercer piso de la calle Schlossfreiheit 1, una de las mejores direcciones berlinesas, al lado de Palacio. Su padre falleció con 53 años en Berlín y su madre con 55 en Riccione, cerca de Rimini (Italia). Nada se sabe de su formación escolar y respecto a sus estudios universitarios se depende de lo que él mismo dice en sus publicaciones. Conforme a ellas, estudió en Berlín durante el curso académico de 1893-1894 en el Seminar

A este respecto cabe mencionar el Centro de Investigación MANES (Manuales Escolares http://www.centromanes.org) cuya colección de material escolar se encuentra en Madrid en la biblioteca de la UNED [consulta: 28 de julio de 2020].

Este se hizo famoso por sus colecciones de cuentos infantiles y más tarde fundó la editorial Calleja, que en el cambio del s. xIx al xx fue toda una institución en el mercado editorial de libros escolares en España.

$4 \quad$ Véase Lombardero Caparrós (2017) que presenta una amplísima colección de obras basadas en el método de Ahn y una colección de parodias y sátiras relacionadas con este método.

“«Es tu primo más alto que mi hermana?», preguntan los periodistas. «No», responde la vicepresidenta, «pero el jardín de mi tía es más grande que la granja de tu abuelo»” (Fernández Briceño, 2012). Una rápida búsqueda en internet da más resultados tanto en la prensa española como la mexicana:

https://www.elmundo.es/opinion/2014/02/28/5310ec09e2704e92548b4572.html [consulta: 28 de julio de 2020]

https://www.gutierrez-rubi.es/2015/01/07/la-politica-y-el-metodo-ollendorff/ [consulta: 28 de julio de 2020].

https://www.eleconomista.com.mx/opinion/Quien-dijo-guerra-20110118-0005.html [consulta: 28 de julio de 2020].

https://elpais.com/diario/1987/12/23/opinion/567212404_850215.html [consulta: 28 de julio de 2020]. 
für Orientalische Sprachen (SOS), una institución dirigida a estudios de lenguas y culturas de oriente. Tener conocimientos de las culturas orientales y disponer de fiables traductores e intérpretes de lenguas exóticas fue una preocupación constante de todas las potencias europeas. Para entenderse con el imperio otomano a partir del s. XVI se utilizaba la figura de jóvenes y adolescentes - los Giovanni di lingua, jóvenes de lenguas, jeunes de langue o Sprachknaben (Marizzi, 2020)—. Por razones obvias que tienen que ver con la tardía conformación de una estructura estatal uniforme, el Imperio Alemán se incorporó tarde en esta pugna por conseguir personas bien formadas y fiables para la toma de contacto con las culturas orientales. Las fechas de creación de las distintas escuelas para el estudio de las culturas orientales son: Francia con el Lycée Louis-le-Grand en 1700, Italia con el Collegio dei Cinesi en 1732 y Austria con la Kaiserlich-königlichen Akademie für orientalische Sprachen fundada por María Teresa en 1754. El más tardío, que data tan solo de 1887, fue en el Imperio Alemán, el Seminar für Orientalische Sprachen, ${ }^{6}$ donde dice haber estudiado Ratti.

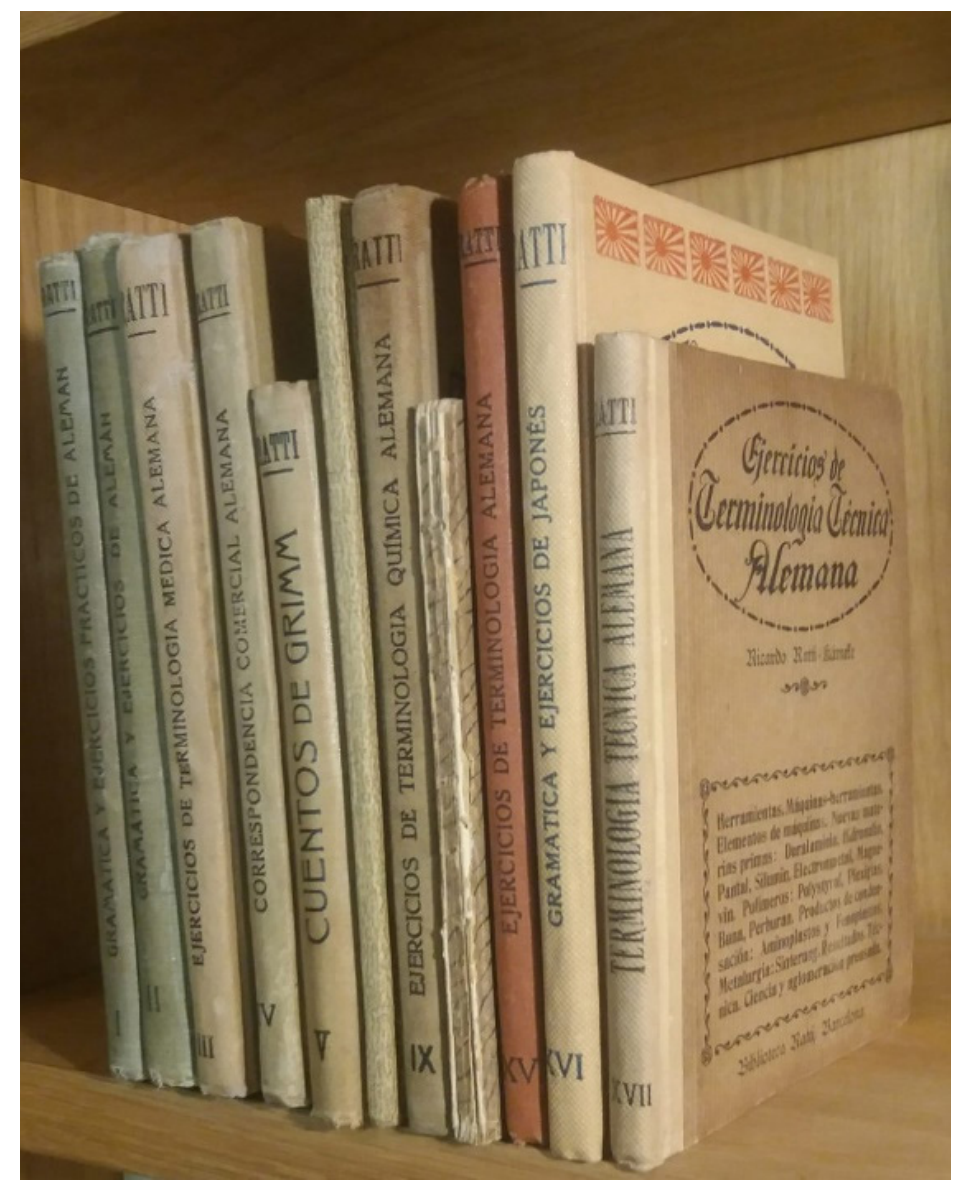

Imagen 1. Biblioteca Ratti (17 tomos). Fuente: colección particular

En los años siguientes se trasladó a Italia, a Nápoles, y según afirmó años más tarde se licenció en japonés con Giulio Gattinoni, el primer catedrático de esta lengua en Italia. ${ }^{7}$ Recientemente se ha podido averiguar en los archivos del Regio Istituto Orientale de Nápoles que Ratti sí estudió chino y ruso en este centro en el curso académico 1903$04 .{ }^{8}$ En estos archivos no consta, sin embargo, que obtuviera el título en japonés a pesar de que sí redactó, o al menos publicó, en 1942 un manual de este idioma (Ratti, 1942) que está registrado con el número XVI entre los volúmenes de la Biblioteca Ratti y que se reimprimió en 1956.

En 1908 consta oficialmente que está establecido en Barcelona, ${ }^{9}$ donde en julio de este año se casó en el Consulado alemán con Agnes Caroline Gertsch (1864-1923), a la que debe de haber conocido en Nápoles, ya que en las amonestaciones (Aufgebot), publicadas en La Vanguardia del 10 de Julio de 1908, se halla la filia-

\footnotetext{
El afán prusiano por ocupar el "Platz an der Sonne” (puesto bajo el sol) que le correspondía conforme a su visión imperialista del devenir de la política internacional se topó con el inconveniente de que el canciller Bismark no contó con fiables traductores del turco en la conferencia de Berlín de 1878 en la que las potencias europeas se proponían la ordenación territorial del sudeste de Europa.

7 "En memoria de Guilio Gattinoni, [...], primer profesor (MCMIV) de lengua japonesa del Regio Instituto Oriental de Nápoles, de cuya mano recibí el primer Diploma de Licenciado en Lengua Japonesa que aquella sabia institución concedió" (Ratti, 1942, II).

8 Los datos están en las memorias del Istituto Universitario Orientale (1904) y Ratti consta con el número de orden 218 en el Elenco nominativo degli alumni iscritti per l'anno 1903-1904.

9 Esto se deduce de la inscripción en el padrón municipal de Barcelona de 1930 en la que consta que el "Sr. Ricardo Ratti Kameke de 57 años de edad, nacido en Alemania, residía en Barcelona, c/ Fontanella $11,2^{\circ}, 1^{\mathrm{a}}$, hacía ya 22 años".
} 
ción de ella: hija de Christian Gertsch y Anna Maria Studer de Gertsch, nacida en Nocera, cerca de Nápoles. Caroline Gertsch tenía 44 años cuando se casó con Ratti en 1908 y falleció sin tener hijos en enero de 1923; Ratti figura en el padrón de Barcelona de 1930 como viudo, empadronado en la calle Fontanella y editor de sus obras propias.

En los años 20 ejerció como profesor de alemán en la Escuela Superior para la Mujer de Barcelona, fundada por Francesca Bonnemaison Farriols (1872-1949), de la que junto con otros fue despedido en el año de 1930 por razones que no se especifican..$^{10}$ Respecto a sus afinidades y actividades políticas en estos años no se sabe nada en concreto, pero entre los años 1936 y 1939 estaba en Bonn y Berlín. Esto hace suponer que la realidad político-social de la Barcelona republicana no le agradaba o que tenía que ausentarse. En Bonn estuvo en contacto con Paul Kahle (1875-1964), el renombrado director del Orientalisches Institut de la Universidad y en Berlín trató con el muy conocido japonólogo Clemens Scharschmidt (1880-1945), director de la Auslandshochschule der Universität Berlín (el viejo Orientalisches Seminar, en el que dice haber estudiado). ${ }^{11}$ A su vuelta en 1939 no parece tener problemas con el nuevo régimen fascista. Al contrario: en 1942 obtuvo una plaza definitiva como profesor de lengua (se supone que alemana) en la Escuela Social de Barcelona (BOE, 1942, p. 8916), que estaba adscrita al Ministerio del Trabajo, y sus obras editadas antes del 36 se seguían publicando. No fueron censurados por la Comisión dictaminadora de Libros de texto ${ }^{12}$ un instrumento de control de material didáctico que los golpistas habían establecido ya en abril de 1938, es decir, un año antes de imponer su régimen. La educación como reguladora de las mentalidades e ideologías no escapa nunca de ningún régimen totalitario.

No constan documentos de Ratti en los que se expresara en catalán, de lo cual se deduce que su socialización en Barcelona debe de haber tenido lugar en castellano exclusivamente. No hay rastros de lengua catalana en sus obras, como tampoco de su paso por Italia quedan vestigios -una gramática del italiano, por ejemplo- si exceptuamos el único italianismo que sale, de modo bastante descontextualizado, en el volumen dos de su gramática general de 1917: "Riepilogando [el subrayado es mío, BM] en parte lo ya dicho" (Ratti, 1917a, p. 37).

El 19 de diciembre de 1945 falleció a la edad de 72 años por un síncope cardíaco en el dispensario de Hostafrancs. Se había casado de nuevo: en el certificado de defunción se menciona su segunda esposa: Josefa Barrot Rodríguez.

Tampoco de este segundo matrimonio tuvo hijos. Pero uno de sus manuales de medicina, que se encuentra en la colección particular del autor de este artículo, lleva una dedicatoria a un médico firmada con fecha del 8 de enero de 1953 por P. Ratti. Una circunstancia que da lugar a la pregunta por la persona que se quedó con los derechos de sus obras y que seguía reeditándolas, como en el caso del manual del japonés cuya reedición tuvo lugar en el año de 1956 (11 años tras su muerte).

\section{LAS INNOVACIONES PRESENTES EN LAS OBRAS DE RATTI}

En el s. XIX empezaron a propagarse, como ya se mencionó más arriba, una serie de sistemas de enseñanza de idiomas, importados sobre todo desde Alemania, ${ }^{13}$ llamados "métodos", que siguen básicamente lo que se ha venido a llamar el Método gramática-traducción. ${ }^{14}$ Entre ellos están, para mencionar los más conocidos y por orden cronológico, los de Meidinger ${ }^{15}$ Ahn, ${ }^{16}$ Ollendorf,,${ }^{17}$ Gaspey-Sauer, ${ }^{18}$ Fábregas, ${ }^{19}$ Robertson, ${ }^{20}$ Berlitz, ${ }^{21}$ Dr. Doppelheim ${ }^{22}$ y la

10 Noticia en La Vanguardia, miércoles, 13 agosto 1930, página 10, "Profesores destituidos de la Escuela Superior de la Mujer" donde figura como "natural de Berlín, licenciado en lenguas modernas por el "Orientalische Seminar, de la Universidad de Berlín” http://hemeroteca.lavanguardia. com/preview/1930/08/13/pagina-10/33212922/pdf.html [consulta: 28 de julio de 2020].

11 "No quiero terminar estos renglones sin dar las gracias al Prof. Dr. Kahle, Director del Orientalische Institut de la Universidad de Bonn, al Prof. Dr. Scharschmidt, Director de la Auslandshochschule [antiguamente Orientalisches Seminar] de la Universidad de Berlín, al Sr. Kölling, jefe de la Sección Oriental de la Imprenta del Reich [Reichsdruckerei] Berlín, al Consulado General del Japón en Barcelona, y a la distinguida y culta pintura Doña Toye Kurimoto, que sucesivamente, y especialmente durante los tres años de mi estancia en Bonn y Berlín (1936/1939), me asistieron en mi trabajo con sus valiosos consejos" (Ratti, 1942: VIII).

12 El Archivo Central de Educación del Ministerio de Educación, Cultura y Deporte (ACME, 2016) conserva en su sede de Alcalá de Henares una colección de 642 libros de textos examinados por dicha Comisión dictaminadora y el Archivo General de la Administración (AGA) custodia las actas de la Comisión dictaminadora, transferidas en el año 1981, con las signaturas ACME 9.839-9.840 y número de registro de entrada AGA 538. Véase también Diego Pérez (2000).

13 Véase al respecto la introducción de Alcalde Mato (2011)

14 Véase acerca del término Corvo Sánchez (2012, p. 148).

15 Johann Valentin Meidinger (1756-1822) es considerado el primer autor mundialmente reconocido con un sistema de enseñanza de lenguas (en su caso del francés)

Véase el ya mencionado estudio de Lombardero Caparrós (2017).

17 Sobre las adaptaciones didácticas realizadas por Benot al Método Ollendorf es de suma importancia el estudio de Villoria (2009).

Estuvo presente en muchos ámbitos geográficos. Véase, para al caso de Portugal, Kemmler y Corvo (2019).

Este fue creado por Sebastián Fábregas (¿?-1854). Véase Fernández Menéndez (2011).

20 Theodore Robertson (i. e. Pierre-Charles-Théodore Lafforgue, 1803-1871) fue un lexicógrafo francés y profesor de inglés. Su método fue introducido en España en 1850 por el venezolano Pedro José Rojas (1818-1874) que adaptó el libro de Robertson al español (Corvo Sánchez 2012). Véase además la entrada Robertson de Suso López en el repertorio del Grup de Recerca en Lingüística Aplicada de la Universitat Rovira y Virgili (http://www.grelinap.recerca.urv.cat/ca/projectes/diccionario-historia-ensenanza-frances-espana/entradas/206/robertson-theodoreparis-1803-1871 [Consulta: 28 de julio de 2020]. También hay al menos dos gramáticas alemanas que siguen el "método Robertson", una de Martin Wilhelm Brasch, Hamburgo 1856, y otra de Luis María Brugada y Panizo, Barcelona 1897 (véase Marizzi et al., 2018, 28 y 55).

21 Más información sobre Maximilian Delphinus Berlitz (1852-1921) y su conocidísimo emporio de enseñanzas de idiomas p. e. en la Biblioteca Virtual de Filología Española (BVFE) https://www.bvfe.es/es/autor/9348-berlitz-maximilian-delphinus.html [Consulta: 28 de julio de 2020 ].

22 Aparte de un manual para aprender la lengua alemana Pelayo Vizuete y Picón (1872-1933) publicó bajo el nombre comercial Método Doppelheim en la editorial Sopena (Barcelona) a principios del s. Xx una serie de manuales del inglés, francés y del italiano. 
denominación se popularizó tanto que ya en el s. XIX muchos manuales y obras de enseñanza de idiomas se llamaron simplemente Metódo de...

Aunque Ricardo Ratti-Kámeke nunca utilizó el nombre de método para referirse ni a su modo de enseñar la lengua alemana ni a sus manuales, se puede destacar que su sistema de aprendizaje significó un gran cambio en el panorama de los manuales de idiomas modernos.

Para las distintas lenguas había, pues, bastantes sistemas de aprendizaje como los arriba mencionados, escritos por varios autores que dieron su nombre a $s u$ método y a la vez había muchos autores que tenían manuales para varios idiomas. ${ }^{23}$ La destacable novedad de los manuales de Ratti —aparte de seguir una novedosa organización interna del libro - residió en la uniformidad de la presentación y a la vez en una clara línea editorial en cuanto a contenidos. Respecto a lo físico, en el plano material se usaron casi siempre las mismas dimensiones [200 x 130], el mismo tipo de cubierta (tapas) y también el mismo tipo de lomo en la mayor parte de los 17 volúmenes que componen su colección, la Biblioteca Ratti (véase imagen 1). A mediados del s. xx otras colecciones y autores siguieron su modelo: entre ellos series tan conocidas como los Manuales Moll ${ }^{24}$ de Francisco de Borja Moll Casanovas (1903-1991), los Métodos Kucera ${ }^{25}$ de Enrique Kucera (St. Gallen, Suiza 1905-Barcelona 1959) y la Biblioteca Lingüistica ${ }^{26}$ de Johann Rauter Schurian (Villach, Austria 1917-Barcelona ¿?) que deberían estudiarse más a fondo.

Richard Ratti-Kámeke fue uno de los primeros innovadores entre los autores de manuales de idiomas en la España de los primeros decenios del s. xx y su gran éxito comercial se inició en 1916 con la primera parte de su método de alemán Gramática y ejercicios prácticos de alemán (Ratti, 1916), que constituye el tomo inicial de su Biblioteca Ratti.

Ya la concepción editorial de su Biblioteca Ratti constituyó, pues, una innovación por reunir en un sello la publicación de manuales prácticos de varios idiomas distintos. En su editorial se publicaron en la primera mitad del s. Xx un total de 17 obras para aprender cuatro idiomas vivos: 10 para estudiar alemán, 5 para el inglés, uno para el francés y otro para el japonés. Respecto al reparto por idiomas, la Biblioteca Ratti se encuentra en una posición diametralmente opuesta a la práctica de su tiempo y refleja el aumento del alemán como lengua de la ciencia. Luego está el inglés, un idioma menos estudiado en la España de esta época, pero en la editorial de Ratti es más frecuente que el francés y se estudia por ser la lengua de la tecnología, de la industria textil y del comercio. ${ }^{27} \mathrm{E}$ francés, la lengua extranjera viva tradicionalmente más estudiada en España, ${ }^{28}$ está representado con una sola obra en la Biblioteca Ratti. Y se trata de un libro que, en realidad, es un subproducto de una publicación que ya existía en su catálogo para lengua alemana, el Preguntón Ratti. Ejercicios de conversación alemana (Ratti, 1920a) (véase imagen 2). Es decir: tanto el Preguntón Ratti. Ejercicios de conversación francesa (Ratti, 1920b) como el Preguntón Ratti. Ejercicios de conversación inglesa (Ratti, 1920c) se vale de la misma composición tipográfica que la versión alemana — con ilustraciones en tres colores (tricromías) - y en la versión francesa e inglesa se cambia nada más que el idioma de la parte de texto. De este modo salen tres versiones de la misma obra pensada para ser utilizada como material adicional para ejercicios de conversación en alemán, inglés y francés.

La concepción metodológica completamente nueva de la enseñanza de idiomas de Ratti se atisba ya en los dos primeros volúmenes de su colección. Orientados a la enseñanza generalista del alemán, contienen ya los primeros intentos de alejarse del tradicional método de gramática-traducción. En el primer volumen de 1916 —aún ordenado por clases de palabras - y en la ampliación al sistema verbal en el segundo (1917) se ponen ejercicios prácticos en las

23 Entre los primeros autores de manuales de varias lenguas cabe destacar para el s. XIX al monje agustino Johann Georg Braun (Gründels/Isny 1828-Santoña 1875) que tiene en su haber gramáticas del alemán, griego, hebreo, inglés y español. Entre los autores españoles están, junto al ya mencionado polígrafo Eduardo Benot, el orientalista y académico de la RAE Francisco García Ayuso (1835-1897), alumno de Braun en el seminario del Escorial. Fue autor de una vasta obra filológica, entre ella manuales del alemán, árabe, francés e inglés. A partir al menos de 1871 dirigió y regentó García Ayuso, que por razones de prestigio solía firmar como D. F. G. Ayuso (Doctor Francisco García Ayuso), una Academia de Lenguas en Madrid, en la que se impartían diecinueve idiomas antiguos y modernos - la mayoría a cargo del propio García Ayuso.

24 Los Manuales Moll para el estudio de las Lenguas Modernas se publicaban desde 1938 en Palma de Mallorca en la Editorial Moll. Para una primera información sobre la "aventura editorial de un filólogo" y su empresa que empezó en 1934, véase el correspondiente dossier "Francesc de B. Moll” en Anthropos (número 44, año 1984) y para información actual de la editorial reflotada en 2015 por la Institució Francesc de Borja Moll.

25 Los manuales de la Editorial fundada por Heinrich (Enrique) Kucera, establecido en Barcelona desde mediados de los años 30 del siglo pasado y propietario de un academia de idiomas, ofrecían el aprendizaje del inglés, francés, alemán, portugués y español para extranjeros según el "Método Kucera". También tenía ya Sprachführer para turistas ingleses, franceses y alemanes siguiendo en muchas ocasiones la misma estructura y las mismas ilustraciones y cambiando solamente de idioma, como ya lo hizo Ratti. El programa de la editorial Kucera era muy amplio y llegó a abarcar a más de 300 títulos. El logo es la imagen de una persona cincelando en una roca dos libros como símil de la cultura. Sus manuales de las diversas lenguas conocieron muchas ediciones y reimpresiones hasta el cese de la actividad editorial en los años 60 del siglo pasado. Una parte de la labor de Enrique Kucera y su editorial está recogida en Bascuñana (2016).

26 La Biblioteca Lingüística del austriaco Johann Rauter-Schurian era otra colección de manuales de inglés, francés, italiano, alemán y latín de una firma barcelonesa, en este caso la Editorial Rauter con sede en Balmes $25,2^{\circ}, 1^{\mathrm{a}}$. Tuvo una gran persistencia en cuanto a las ediciones de sus manuales que no solamente contenían obras para la enseñanza de idiomas sino otros muchos libros de texto de las más variadas asignaturas. Fuera de la Biblioteca Lingüistica llegó a publicar varios diccionarios especializados como el Diccionario alemán-español de terminología química, farmacéutica y bioquímica de José Chabás López (1954), siguiendo en cierto sentido la línea editorial de Ratti en relación con lenguas especializadas. Según datos de archivos austriacos, el dueño de la editorial estaba muy activo en sus actividades de propagación de la cultura, el turismo y la política austriaca en Barcelona (Müller et alii, 2016, 34f.).

27 Véase al respecto, entre otros: Martín-Gamero (1961) y más recientemente Lombardero Caparrós (2019).

28 De nuevo remitimos a la ya citada página de la Rovira y Virgili. 
cuatro destrezas: leer, escribir, escuchar y hablar. Las cuestiones sintácticas aparecen en anotaciones a los distintos ejercicios, no en apartados teóricos aislados. Solamente al final del segundo volumen se da un escueto resumen de la sintaxis elemental en dos páginas.

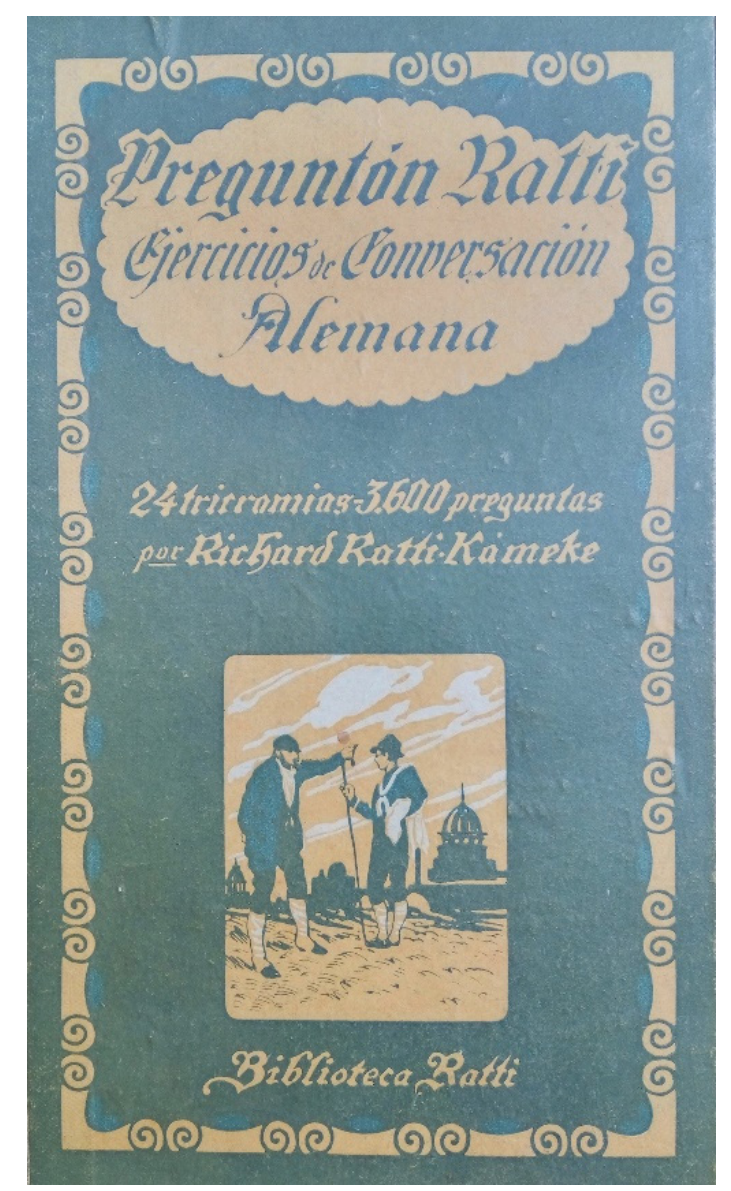

Imagen 2. Preguntón Ratti. Ejercicios de conversación alemana (Biblioteca Ratti, V).

Fuente: colección particular

Donde, sin embargo, no destaca Ratti es en la actualización de sus manuales: el primer volumen de su Gramática y ejercicios prácticos de alemán salió en 1916 en plena Primera Guerra Mundial y no cambia hasta la octava edición de 1941. Ni en el año de su primera edición de 1916 reflejó la realidad de su tiempo: no se nota que Europa está en guerra y se puede viajar en tren desde Múnich a Venecia. El segundo volumen cambia algo, salió en 1917 y ya tuvo en cuenta los acontecimientos bélicos: "Se anuncia [es wird gemeldet] que la ciudad [de] Bukarest [sic, B.M.] ha sido ocupada por nuestras tropas" (Ratti, 1917a, p. 62). El uso del posesivo "nuestras tropas" ya reflejaba la posición que adopta en el conflicto.

Como novedad y como refuerzo de los manuales generalistas salieron en estos años métodos con ejercicios pensados para fomentar la comprensión lectora. En consonancia con el ideal de la formación humanística, esta serie se inició con textos literarios. Ya en 1918 apareció la edición de los cuentos más conocidos de los Hermanos Grimm (Cuentos de Grimm. Ejercicios de lectura alemana, Ratti 1918), en una edición especialmente atractiva (véase imagen 3).

En España los cuentos de los Grimm se venían publicando en su lengua original ya desde 1864 en manuales de lengua alemana y en esto hay que tener en cuenta que la primera traducción al español salió solamente un año antes -en 1863- y no en España, sino en París. Desde este año de 1864 suelen formar parte de los manuales de enseñanza teórico-práctica, en los que la primera parte está dedicada a la gramática tradicional según el modelo de las gramáticas latinas, y que se completa con una segunda parte que contiene un apartado de fragmentos selectos para la aplicación de las destrezas adquiridas. En la mayoría de los casos esta se restringía sobre todo a la traducción directa, es decir, al método de gramática-traducción. A partir de los inicios del s. xx los cuentos de los Grimm también formaron parte de antologías de textos alemanes - llamadas Versiones, Libros de lectura, Lecturas graduadas o Ejercicios de lectura - en las que se ofrecía material adicional para mejorar la comprensión lectora (Marizzi, 2014).

El tomo de Ratti es, por tanto, uno de los primeros manuales concebidos con el fin específico de integrar la explicación gramatical, la comprensión lectora y la transmisión de aspectos de la cultura de los países de la lengua estudiada, en este caso el alemán. 


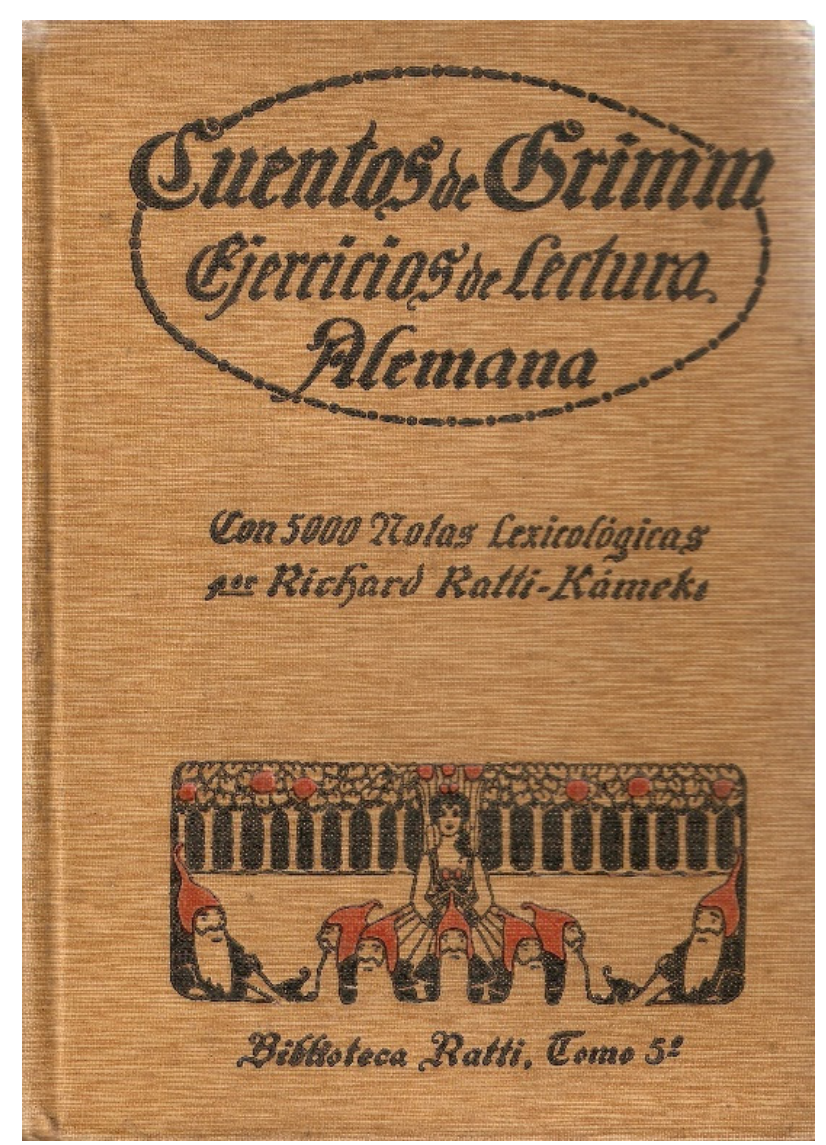

Imagen 3. Cuentos de Grimm. Ejercicios de lectura alemana (Biblioteca Ratti, V).

Fuente: colección particular

Con la misma intención apareció años más tarde Poesías de Goethe (Ratti, 1932), un volumen que reúne 24 poemas de este autor completados con la transcripción de las composiciones de varios músicos inspirados en la lírica de Goethe. En el prólogo indica Ratti que la música es "el único lenguaje admisible para la traducción de las poesías de Goethe, son tan finas, tan delicadas como la pelusilla de las alas de las mariposas; no permiten manejos violentos" (Ratti, 1932, III). Para poder alcanzar alguna comprensión orientativa añade, sin embargo, anotaciones léxicas que llegan a alcanzar el grado de traducciones palabra por palabra. Otras observaciones ofrecen información sobre el momento de la vida de Goethe en el que escribió las poesías y sobre los compositores que le pusieron música al texto.

Una innovación muy llamativa fue el enfoque que en las obras de Biblioteca Ratti se dio al alemán como lengua que sirve para comunicar y difundir los avances científicos. En este aspecto Ratti está en línea con casi todos los autores de gramáticas alemanas que le preceden — aparte de los ya mencionados Braun y García Ayuso, especialmente Julio Kühn (1813-1854) y Carlos Fernández Lletor de Castroverde, (Iznalloz, Granada, ¿?-1890)— pero a Ratti le caracteriza la particularidad con que lo llevó a cabo desde los primeros tomos de su colección de manuales y el hecho de que este enfoque aparece ya en el título de sus obras.

\section{ALEMÁN COMO LENGUA DE LA CIENCIA}

En cuanto a manuales dedicados a ciencias están los Ejercicios de terminología médica alemana (Ratti, 1917b) (véase imagen 4), Ejercicios de terminología química alemana (Ratti, 1923a) y Terminología alemana de derecho $y$ de ciencias económicas y sociales (Ratti, 1936). En la introducción a todas ellas, Ratti refleja las experiencias del estudiante de idiomas que:

después de haber discurrido largamente por las páginas áridas de la gramática, difícilmente pueden desentrañar el sentido del primer libro de técnica médica alemana de que quieren servirse, por desconocer, con la seguridad que se requiere, el léxico y la sintaxis especiales de la clase de ciencia que desean consultar (Ratti, 1917b, III).

Cabe destacar que en España pasaron treinta años hasta que se llevaran a la práctica las ideas contenidas en el panfleto programático del filólogo Wilhelm Viëtor (1850-1918) Der Sprachunterricht muss umkehren 
(Viëtor, 1886) y que el movimiento de la Reforma en la enseñanza lingüística tuviera ciertas consecuencias prácticas como en este caso. ${ }^{29}$

Si bien Ratti publicó manuales dedicados a suplir este déficit, también hay que añadir que en ello el autor recurre solamente a una ampliación del viejo sistema de gramática-traducción; no se practican diálogos y el único objetivo es la comprensión lectora en la lengua meta. Como novedad cabe destacar que implícitamente el autor presupone que el alumno ya tiene conocimientos básicos, y por ello no expone de manera ordenada los rudimentos de la gramática alemana y se limita a la compilación de un corpus de textos de la especialidad en cuestión. Estos textos son glosados con un vocabulario y comentados en lo tocante a accidentes gramaticales particulares del alemán científico.

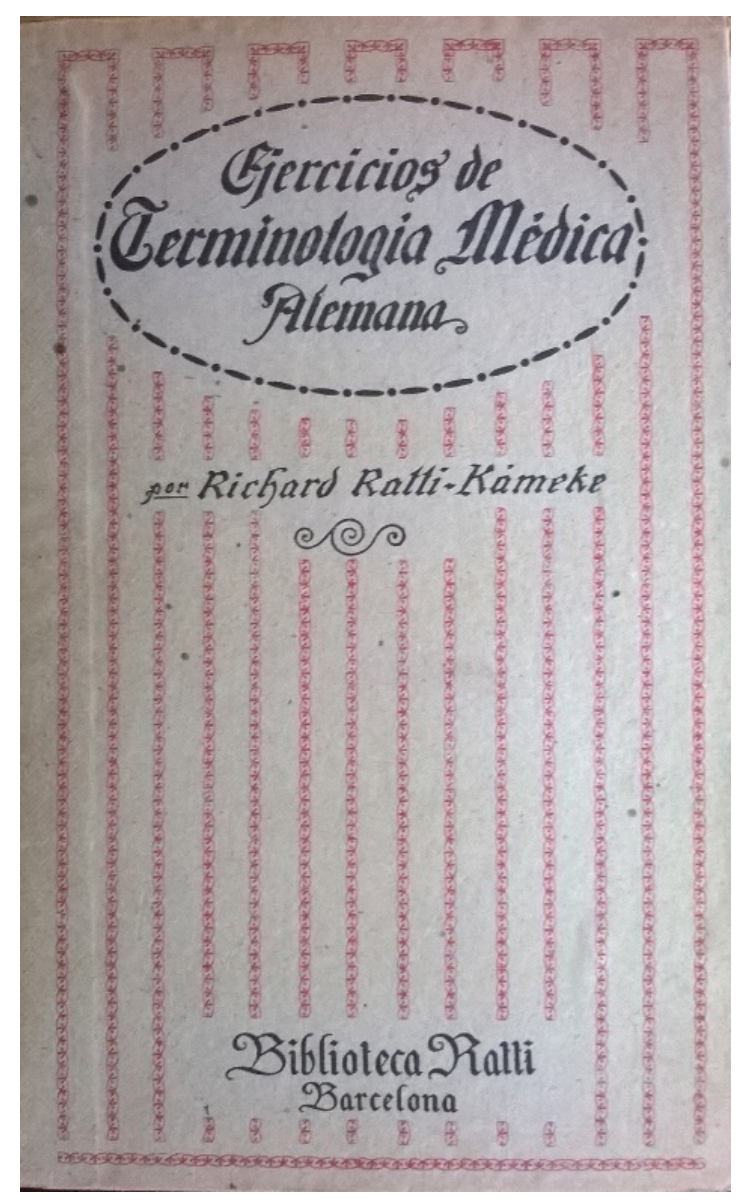

Imagen 4. Ejercicios de terminología médica alemana, (Biblioteca Ratti, III).

Fuente: colección particular

En cuanto al contenido, trata los temas básicos de cada especialidad y se denota una progresión que se corresponde con la estructura de una carrera universitaria. Ya hemos mencionado que el vocabulario introduce las palabras básicas de los distintos textos. En Ejercicio aparece un texto en alemán que el autor ha redactado expresamente para cada lección y que tenía que ser pasado al español por el alumno. Como Tema ${ }^{30}$ hay un texto en español para traducirlo al alemán. Un fragmento procedente de un libro de la especialidad llamado Lectura cierra cada unidad. Los tres tipos de texto vienen acompañados de un aparato de notas a pie de página en los que se comentan cuestiones de formación de palabras y problemas sintácticos. Todas estas lecciones siguen el mismo patrón y exigen en todo caso la traducción directa e inversa. La gran novedad es que estos textos eran textos de la especialidad.

Queda claro que en estos manuales de ejercicios enfocados a la lengua específica de la ciencia —un concepto completamente nuevo en la España de principios de s. $\mathrm{xx}$-, el lenguaje científico se caracteriza por dos elementos básicos: la terminología y la sintaxis específicas. No se trataba de transmitir a los alumnos nuevos conocimientos de la especialidad, sino de presentarles en alemán los nombres y conceptos que en un principio ya conocían y explicar las estructuras necesarias para comprender los textos alemanes.

29 Aunque en honor a la verdad hay que decir que Viëtor sí estaba en contacto epistolar a finales del s. XIX con Fernando Araujo Gómez (18571914/1915?), catedrático de secundaria de francés y alemán en Salamanca, Toledo y Madrid y autor de una voluminosa gramática generalista alemana (1897) que sin embargo parece que no llevaba a la práctica las ideas de la Reforma lingüística en la enseñanza del alemán.

30 Esta parte práctica de los manuales son, desde las gramáticas de Meidinger (véase nota 13), constitutivas del método de gramática-traducción. Véanse las apreciaciones de Klippel (1994, p. 36) y Corvo Sánchez (2011, p. 187). 
En este aspecto hay una gran diferencia con los actuales manuales y conceptos didácticos dedicados a las lenguas específicas: Ratti dirigía sus libros de texto a un público de científicos en ejercicio de la profesión, pero no recurría en sus enseñanzas a los conocimientos previos del alumnado como sería costumbre hoy en día. Especialmente la enseñanza del alemán como lengua de la ciencia a científicos españoles debería incorporar desde el principio estos conocimientos. Por el contrario son frecuentes las ocasiones en las que se explican palabras que cualquier especialista entiende sin más. Véanse términos médicos en latín como "endometritis decidualis gonorrheica" que pasa a ser en español "endometritis decidual gonorreica" (Ratti, 1917b, p. 149). A un nivel de conocimiento menos elevado se entiende sin más la palabra "Praxis", pero Ratti lo "traduce" en una nota en el manual de química (Ratti, 1923a, 76n27). Algo parecido ocurre en el volumen de derecho: pone "Die kritische Methode -El método crítico-" (Ratti, 1936, p. 8) o en el libro de medicina "die kontraktile Sustanz —La substancia contráctil—" (Ratti, 1917c, 10n5).

La falta de un método para automatizar la comprensión de la formación de palabras en alemán se manifiesta en que los más importantes sufijos aparecen ya en las primeras lecciones de sus manuales, pero en todos se vuelven a explicar estas estrategias derivativas una y otra vez. Esta postura didáctica hoy en día sería diametralmente opuesta a conceptos ampliamente asumidos como la autonomía del alumno y el trabajo individual que tienen que realizar los estudiantes por su cuenta para hacerse con el vocabulario.

En relación con la terminología específica se observa en Ratti una posición ambigua. Por un lado ya se comentó que no recurre a los conocimientos previos de los alumnos; recordamos que explica, por ejemplo, "Praxis" y "die kontraktile Substanz". Por otro lado, se presenta un anacronismo lingüístico en versiones alemanas hoy en desuso como: "Geldmann" para Kapitalist (Ratti, 1936, p. 107), "drahtlich" para telegraphieren y "Drahtbericht", "Drahtung" y "Drahtantwort" para Telegramm (Ratti, 1936, p. 118).

El hecho de que solía reimprimir ${ }^{31}$ sus obras sin corregir las pocas erratas que tienen ni introducir mejoras que tuvieran en cuenta los cambios políticos y sociales se observa en una de las cartas comerciales del manual Ejercicios de correspondencia comercial alemana. En la segunda edición de 1930 figura el mismo encabezamiento de una carta comercial que en 1917 y de este modo sigue poniendo el nombre "Deutsch Südwestafrika" (Ratti, 1917c, p. 83) para designar un territorio que ya en 1916 fue ocupado por los británicos. Continúa, por lo tanto, rememorando esta antigua colonia alemana (hoy Namibia) que tras la Primera Guerra Mundial pasó a formar parte de Sudáfrica.

Como colofón y políticamente hablando se puede decir que Ratti asumió primero el punto de vista de la Alemania imperial, como se comprueba en el pasaje citado arriba, y luego el de la Alemania nazi, ya que en unos ejercicios de la Terminología alemana de derecho y de ciencias económicas y sociales aparecen frases como: "[...] und der durch das nationalsozialistische Strafrecht geschaffene Tatbestand der Rassenschande sind staatsgefährdende Delikte" ([...] y el hecho de ofensa a la raza, creado por el derecho penal nacionalsocialista, son [sic] delitos que ponen en peligro al estado, trad. de BM) (Ratti, 1936, p. 50). También habla de la "nationale Revolution" (Ratti, 1936, p. 115) y dice que "politische Verbrecher verbüßten früher ihre Strafen in Festungen, heute in Konzentrationslagern" (antes, criminales políticos cumplían sus penas en prisiones militares, hoy en campos de concentración, trad. de BM) (Ratti, 1936, p. 51).

Esto no disminuye, sin embargo, sus méritos como innovador en cuanto a los métodos didácticos y estilísticos que utilizó.

\section{CONCLUSIÓN}

Es de destacar el papel innovador de Richard Ratti-Kámeke especialmente en cuanto al tratamiento de los tipos de textos que presentan el alemán como lengua de la ciencia. Sin embargo, también queda claro que aún falta mucha investigación sobre la estrategia comercial y el devenir de las casas editoriales mencionadas y líneas editoriales posteriores, sobre todo en Barcelona. Por ejemplo, cabe mencionar la Editorial Rauter en cuanto a materiales de alemán como lengua de la ciencia (Chabás López, 1954). También falta información sobre los posibles herederos de RattiKámeke y el responsable de la reedición de varios de sus manuales.

\section{BIBLIOGRAFÍA}

Alcalde Mato, Nuria (2011). Principales métodos de enseñanza de lenguas extranjeras en Alemania. Revista de Lingüistica y Lenguas Aplicadas, 6, 9-24. Https://doi.org/10.4995/rlyla.2011.878.

Anthropos. Boletín de Información y Documentación (1984). Dossier dedicado a Francesc de B. Moll. Anthropos, 44, $7-21$.

Archivo Central de Educación del Ministerio de Educación, Cultura y Deporte (ACME) (2016). Comisión Dictaminadora de Libros de Texto. Https://www.educacionyfp.gob.es/ca/dam/jcr:133dc858-fd48-49f9-a482-adc16b8fb595/librosdetexto.pdf.

Chabás López, José (1954). Diccionario alemán-español de terminología química, farmacéutica y bioquímica. Barcelona: Editorial Rauter.

31 El número de edición de la obra en cuestión no se refleja, por esta razón, en las referencias bibliográficas de las citas en el cuerpo del texto; número de edición que, sin embargo, sí se pone en la bibliografía final. 
Corvo Sánchez, María José (2011). Historia y tradición en la enseñanza y aprendizaje de lenguas extranjeras en Europa (VIII): siglos XVII y XVIII. La enseñanza moderna y las tradiciones nacionales. Babel A.F.I.A.L.: Aspectos de Filología Inglesa y alemana, 20, 163-191.

Corvo Sánchez, María José (2012). Historia y tradición en la enseñanza y aprendizaje de lenguas extranjeras en Europa (IX): Siglo XIX, hacia el presente de la didáctica de lenguas modernas. Babel A.F.I.A.L.: Aspectos de Filología Inglesa y Alemana, 21, 137-166.

Diego Pérez, Carmen (2000). Dictamen y dotación de libros de texto desde la guerra civil hasta la creación del Consejo Nacional de Educación. Historia de la Educación, 19, 293-309. Https://revistas.usal.es/index.php/0212-0267/article/ view/10802/11200.

Fernández Briceño, Jesús (2012, julio 29). Método Ollendorff. La Gaceta (Madrid). Https://www.jesusfb.com/index. php/2012/07/metodo-ollendorf/.

Fernández Menéndez, María Antonia (2011). Métodos para la enseñanza del inglés durante el siglo xIx y primera mitad del siglo xx. Tonos Digital, Revista Electrónica de Estudios Filológicos, 21. Https://www.um.es/tonosdigital/znum21/ secciones/estudios-12-ensenanza ingles.htm.

García Bascuñana, Juan Francisco (2016). Diccionario de historia de la enseñanza del francés en España (siglos XVI-XX). Http://www.grelinap.recerca.urv.cat/es/projectes/diccionario-historia-ensenanza-frances-espana/.

Grup de Recerca en Lingüística Aplicada de la Universitat Rovira y Virgili. Http:/www.grelinap.recerca.urv.cat.

Institució Francesc de Borja Moll. Http://editorialmoll.cat/botiga/ca/.

Istituto Universitario Orientale (1904). Memorie, Fascicolo I, Anni scolastici 1900-01 a 1903-04. Napoli: Tipografia Melei \& Joele. [Legajo de Semitistica, $\left.n^{\circ} 671\right]$.

Kemmler, Rolf (2019). Die frühesten portugiesischen Deutschlehrwerke (1863-1926). Vila Real: Centro de Estudos em Letras, Universidade de Trás-os-Montes e Alto Douro.

Kemmler, Rolf y María José Corvo Sánchez (2019). The importance of the 'method Gaspey-Otto-Sauer' amongst the earliest Portuguese textbooks of the German language. Language \& History, 63(2), 120-138. Https://www.tandfonline. com/doi/full/10.1080/17597536.2019.1649854.

Klippel, Friederike (1994). Englischlernen im 18. und 19. Jahrhundert: Die Geschichte der Lehrbücher und Unterrichtsmethoden. Münster: Nodus Publikationen.

Lombardero Caparrós, Alberto (2019). Two Centuries of English Language Teaching and Learning in Spain: 1769-. Amsterdam: Amsterdam University Press.

Lombardero Caparrós, Alberto (2017). La influencia del método de Ahn en España en los siglos XIX y Xx a través de una mirada a la prensa histórica. Documents pour l'histoire du français langue étrangère ou seconde, 58-59, 83-101. Https://journals.openedition.org/dhfles/4453.

Martín Gamero, Sofía (1961). La enseñanza del inglés en España. Madrid: Gredos.

Müller, Stefan A., David Schriffl y Adamantios T. Skordos (2016). Heimliche Freunde: Die Beziehungen Österreichs zu den Diktaturen Südeuropas nach 1945: Spanien, Portugal, Griechenland. Wien: Böhlau.

Marizzi, Bernd (2014). Los cuentos de los hermanos Grimm en los manuales de lengua alemana españoles entre 1864 y 1943. En Isabel Hernández y Miriam Llamas (eds.). Los hermanos Grimm en contexto. Reescritura e interpretación de un legado universal (pp. 189-200). Madrid: Síntesis.

Marizzi, Bernd (2020). Die Erkundung des Morgenlandes zu Beginn der Neuzeit: Giovanni di lingua - Enfants de Langues - Jóvenes de lenguas - Sprachknaben [en imprenta].

Marizzi, Bernd, María Teresa Cortez y María Teresa Fuentes Morán (2018). Deutschlernen in Spanien und Portugal. Eine teilkommentierte Bibliographie von 1502 bis 1975 (Fremdsprachen in Geschichte und Gegenwart 19). Wiesbaden: Harrassowitz.

Orden de 15 de octubre de 1942 (rectificada) por la que se nombran, con carácter definitivo, Director, Secretario y Profesores de la Escuela Social de Barcelona. Boletín Oficial del Estado, de 4 de noviembre de 1942, p. 8916. Https:// www.boe.es/datos/pdfs/BOE/1942/308/A08916-08916.pdf.

Ratti-Kámeke, Richard $\left(1916,{ }^{4} 1923,{ }^{6} 1932,{ }^{7} 1935,{ }^{8} 1941\right)$. Gramática y ejercicios prácticos de alemán (Biblioteca Ratti, I). Barcelona: Imp. Modesto Berdós Coll.

Ratti-Kámeke, Richard (1917a, $\left.{ }^{2} 1923,{ }^{3} 1935\right)$. Gramática y ejercicios de prácticos alemán, segunda parte (Biblioteca Ratti, II). Barcelona: Imp. Modesto Berdós Coll.

Ratti-Kámeke, Richard (1917b, ${ }^{2}$ 1921, $\left.{ }^{3} 1931,{ }^{4} 1943\right)$. Ejercicios de terminología médica alemana (Biblioteca Ratti, III). Barcelona: Imp. Modesto Berdós Coll.

Ratti-Kámeke, Richard (1917c, $\left.{ }^{2} 1930\right)$. Ejercicios de correspondencia comercial alemana (Biblioteca Ratti, IV). Barcelona: Imp. Modesto Berdós Coll.

Ratti-Kámeke, Richard (1918, $\left.{ }^{2} 1930,{ }^{3} 1941\right)$. Cuentos de Grimm. Ejercicios de lectura alemana (Biblioteca Ratti, V). Barcelona: Imp. Modesto Berdós Coll.

Ratti-Kámeke, Richard (1920a). Preguntón Ratti. Ejercicios de conversación alemana (Biblioteca Ratti, V). Barcelona: Imp. La Cervantina.

Ratti-Kámeke, Richard (1920b). Preguntón Ratti. Ejercicios de conversación francesa (Biblioteca Ratti, VIII). Barcelona: Imp. La Cervantina. 
Ratti-Kámeke, Richard (1920c). Preguntón Ratti. Ejercicios de conversación inglesa (Biblioteca Ratti, VII). Barcelona: Imp. La Cervantina.

Ratti-Kámeke, Richard (1921). Correspondencia comercial inglesa (Biblioteca Ratti, XI). Barcelona: Imp. Modesto Berdós Coll.

Ratti-Kámeke, Richard (1922). Ejercicios de terminología técnica inglesa (Biblioteca Ratti, XII). Barcelona: Imp. Modesto Berdós Coll.

Ratti-Kámeke, Richard (1923a, ${ }^{2}$ 1943). Ejercicios de terminología química alemana (Biblioteca Ratti, IX). Barcelona: Imp. Modesto Berdós Coll.

Ratti-Kámeke, Richard (1923b). "Practical English”. Gramática y ejercicios prácticos de inglés (Biblioteca Ratti, X). Barcelona: Imp. Modesto Berdós Coll.

Ratti-Kámeke, Richard (1924). Textil english. Ejercicios de terminología textil inglesa (Biblioteca Ratti, XII). Barcelona: Imp. Modesto Berdós Coll.

Ratti-Kámeke, Richard (1932). Poesías de Goethe: ejercicios de lectura alemana, con anotaciones musicales, lexicológicas e históricas (Biblioteca Ratti, XIV). Barcelona: Imp. Modesto Berdós Coll.

Ratti-Kámeke, Richard (1936). Terminología alemana de derecho y de ciencias económicas y sociales (Biblioteca Ratti, Xv). Barcelona: Imp. Modesto Berdós Coll.

Ratti-Kámeke, Richard (1942, $\left.{ }^{2} 1956\right)$. Gramática y ejercicios prácticos de japonés (Biblioteca Ratti, XVI). Barcelona: Imp. Modesto Berdós Coll.

Ratti-Kámeke, Richard (1945). Ejercicios de terminología técnica alemana (Biblioteca Ratti, XVII). Barcelona: Imp. Modesto Berdós Coll.

Suso López, Javier (2009). Apuntes para una historia de la enseñanza de la lengua francesa en España. En Antonio Martínez González (ed.). Historia de las ideas lingüísticas en España. Gramáticos en la España meridional (pp. 162170). Frankfurt: Lang.

Suso López, Javier (2020). Robertson. Http://www.grelinap.recerca.urv.cat/ca/projectes/diccionario-historia-ensenanzafrances-espana/entradas/206/robertson-theodore-paris-1803-1871.

Viëtor, Wilhelm (1886). Der Sprachunterricht muss umkehren. Https://archive.org/details/dersprachunterr02vigoog.

Villoria Prieto, Javier (2009). Innovación en la enseñanza de lenguas: el Nuevo Método de Eduardo Benot. En Antonio Martínez González (ed.). Historia de las ideas lingüisticas en España. Gramáticos en la España meridional (pp. 133159). Frankfurt: Lang. 